\title{
Some fixed point theorems for $(\alpha, \beta)$-admissible Geraghty type contractive mappings and related results
}

\author{
Sumit Chandok ${ }^{1}$
}

Received: 28 October 2014 / Accepted: 8 June 2015/Published online: 27 June 2015

(C) The Author(s) 2015. This article is published with open access at Springerlink.com

\begin{abstract}
In this paper, we introduced the concept of $(\alpha$, $\beta$ )-admissible Geraghty type contractive mappings. Sufficient conditions for the existence of a fixed point for such class of generalized nonlinear contractive mappings in metric spaces are provided. As applications, we derive a fixed point theorem for these contractions whenever the space is endowed with a graph. Some interesting consequences of our theorems are also obtained. The proved results generalize and extend various well-known results in the literature. Some examples are illustrated for the usability of the results.
\end{abstract}

Keywords Fixed point $\cdot(\alpha, \beta)$-admissible $\cdot$ Contraction mappings $\cdot$ Metric space

Mathematics Subject Classification $47 \mathrm{H} 10 \cdot 54 \mathrm{H} 25$

\section{Introduction and preliminaries}

Fixed point theory has gained very large impetus due its wide range of applications in various fields such as engineering, economics, computer science, and many others. It is well known that the contractive-type conditions are very indispensable in the study of fixed point theory and Banach's fixed point theorem [1] for contraction mappings

Sumit Chandok

chansok.s@gmail.com

1 Department of Mathematics, Khalsa College of Engineering and Technology, Punjab Technical University, Amritsar 143001, India is one of the pivotal results in analysis. This theorem that has been extended and generalized by various authors (see, e.g., [2-15]) and has many applications in mathematics and other related disciplines as well.

In an attempt to generalize the Banach contraction principle, many researchers extended the following result in certain directions.

Theorem 1.1 (see, [8]) Let $(X, d)$ be a complete metric space and $T: X \rightarrow X$ be a mapping. Assume that there exists a function $\theta:[0, \infty) \rightarrow[0,1]$ such that, for any bounded sequence $\left\{t_{n}\right\}$ of positive reals, $\theta\left(t_{n}\right) \rightarrow 1$ implies $t_{n} \rightarrow 0$ and $d(T x, T y) \leq \theta(d(x, y)) d(x, y)$ for all $x, y \in X$. Then, $T$ has a unique fixed point.

In 2012, Samet et al. [15] introduced the concepts of $\alpha$ contractive and $\alpha$-admissible mappings and established various fixed point theorems for such class of mappings defined on complete metric spaces. Thereafter, the existence of fixed points of $\alpha$-admissible contractive-type mappings in complete metric spaces has been studied by several researchers (see $[2,11,13,14]$ and references cited therein). In this paper, we introduced the concept of $(\alpha, \beta)$ admissible Geraghty type contractive mappings. Sufficient conditions for the existence of a fixed point for such class of generalized nonlinear contractive mappings in metric spaces are provided. As applications, we derive a fixed point theorem for these contractions whenever the space is endowed with a graph. Several other interesting results for cyclic mappings and ordered metric spaces are also deduced. The proved results using the concept of $(\alpha, \beta)$ admissible mappings generalize and extend various wellknown results in the literature. Some examples are illustrated for the justification of the results.

To start with we give some notations and introduce some definitions which will be used in the sequel. 
Definition 1.1 Let $X$ be a non-empty set, $T: X \rightarrow X$ and $\alpha, \beta: X \times X \rightarrow \mathbb{R}^{+}$. We say that $T$ is an $(\alpha, \beta)$-admissible mapping if $\alpha(x, y) \geq 1$ and $\beta(x, y) \geq 1$ implies $\alpha(T x, T y) \geq 1$, and $\beta(T x, T y) \geq 1$, for all $x, y \in X$.

Definition 1.2 Let $X$ be a non-empty set, $T: X \rightarrow X$ and $\alpha: X \times X \rightarrow \mathbb{R}^{+}$. We say that $T$ is an $\alpha$-admissible mapping if $\alpha(x, y) \geq 1$ and implies $\alpha(T x, T y) \geq 1$, for all $x, y \in X$.

Definition 1.3 Let $(X, d)$ be a metric space, and $\alpha, \beta: X \times X \rightarrow[0, \infty)$. $X$ is $(\alpha, \beta)$-regular if $\left\{x_{n}\right\}$ is a sequence in $X$ such that $x_{n} \rightarrow x \in X, \alpha\left(x_{n}, x_{n+1}\right) \geq 1$, $\beta\left(x_{n}, x_{n+1}\right) \geq 1$, for all $n$, there exists a subsequence $\left\{x_{n_{k}}\right\}$ of $\left\{x_{n}\right\}$ such that $\alpha\left(x_{n_{k}}, x_{n_{k}+1}\right) \geq 1, \beta\left(x_{n_{k}}, x_{n_{k}+1}\right) \geq 1$ for all $k \in \mathbb{N}$ and $\alpha(x, T x) \geq 1, \beta(x, T x) \geq 1$.

Definition 1.4 Let $(X, d)$ be a metric space, and $\alpha: X \times X \rightarrow[0, \infty) . X$ is a $\alpha$-regular if $\left\{x_{n}\right\}$ is a sequence in $X$ such that $x_{n} \rightarrow x, \alpha\left(x_{n}, x_{n+1}\right) \geq 1$, there exists a subsequence $\left\{x_{n_{k}}\right\}$ of $\left\{x_{n}\right\}$ such that $\alpha\left(x_{n_{k}}, x_{n_{k}+1}\right) \geq 1$ for all $k \in \mathbb{N}$ and $\alpha(x, T x) \geq 1$.

Definition 1.5 Let $(X, d, \leq)$ be an ordered metric space, and $\alpha: X \times X \rightarrow[0, \infty) . X$ is an ordered $\alpha$-regular if $\left\{x_{n}\right\}$ is a sequence in $X$ such that $x_{n} \rightarrow x, \alpha\left(x_{n}, x_{n+1}\right) \geq 1$, and $x_{n} \leq x_{n+1}$ then there exists a subsequence $\left\{x_{n_{k}}\right\}$ of $\left\{x_{n}\right\}$ such that $\alpha\left(x_{n_{k}}, x_{n_{k}+1}\right) \geq 1$ and $x_{n_{k}} \leq x_{n_{k}+1}$ for all $k \in \mathbb{N}$ and $\alpha(x, T x) \geq 1$.

Throughout the paper, $F(T)$ denotes the set of fixed points of $T$.

\section{Main results}

We say $\Theta$ be a family of functions $\theta:[0, \infty) \rightarrow[0,1)$ such that for any bounded sequence $\left\{t_{n}\right\}$ of positive reals, $\theta\left(t_{n}\right) \rightarrow 1$ implies $t_{n} \rightarrow 0$.

We say $\Psi$ be a family of functions $\psi:[0, \infty) \rightarrow[0, \infty)$ such that $\psi$ is continuous, strictly increasing and $\psi(0)=0$.

Definition 2.1 Let $(X, d)$ be a metric space, $T: X \rightarrow X$ and $\alpha, \beta: X \times X \rightarrow \mathbb{R}^{+}$. A mapping $T$ is said to be $(\alpha, \beta)$ Geraghty type-I rational contractive mapping if there exists a $\theta \in \Theta$, such that for all $x, y \in X$, following condition holds:

$$
\alpha(x, T x) \beta(y, T y) \psi(d(T x, T y)) \leq \theta(\psi(M(x, y))) \psi(M(x, y)),
$$

where $M(x, y)=\max \left\{d(x, y), d(x, T x), d(y, T y), \frac{d(x, T x) d(y, T y)}{1+d(x, y)}\right.$, $\left.\frac{d(x, T x) d(y, T y)}{1+d(T x, T y)}\right\}$ and $\psi \in \Psi$.

Theorem 2.1 Let $(X, d)$ be a complete metric space, $T$ is self-mapping, $T: X \rightarrow X$, and $\alpha, \beta: X \times X \rightarrow \mathbb{R}^{+}$. Suppose that the following conditions are satisfied: (i) $T$ is an $(\alpha, \beta)$-admissible mapping;

(ii) $T$ is an $(\alpha, \beta)$-Geraghty type-I rational contractive mapping;

(iii) there exists $x_{0} \in X$ such that $\alpha\left(x_{0}, T x_{0}\right) \geq 1$ and $\beta\left(x_{0}, T x_{0}\right) \geq 1$;

(iv) either $T$ is continuous or $X$ is $(\alpha, \beta)$-regular.

Then, Thas a fixed point $x \in X$ and $\left\{T^{n} x_{0}\right\}$ converges to $x$.

Further, if for all $x, y \in F(T)$, with $x \neq y$ such that $\alpha(x, T x) \geq 1, \alpha(y, T y) \geq 1$ and $\beta(x, T x) \geq 1, \quad \beta(y, T y) \geq 1$, then $T$ has a unique fixed point in $X$.

Proof Let $x_{0} \in X$ such that $\alpha\left(x_{0}, T x_{0}\right) \geq 1$ and $\beta\left(x_{0}, T x_{0}\right) \geq 1$. Now, we can construct the sequences $\left\{x_{n}\right\}$ in $X$ by $x_{n}=T^{n} x_{0}=T x_{n-1}$, for $n \in \mathbb{N}$.

Moreover, we assume that if $x_{n_{0}}=x_{n_{0}+1}$, for some $n_{0} \in \mathbb{N}$, then $x_{n_{0}}$ is a fixed point of $T$. Consequently, we suppose that $x_{n} \neq x_{n+1}$ for all $n \in \mathbb{N}$.

Since $T$ is $(\alpha, \quad \beta)$-admissible mapping, $\alpha\left(x_{0}, T x_{0}\right)=\alpha\left(x_{0}, x_{1}\right) \geq 1, \quad \alpha\left(T x_{0}, T x_{1}\right)=\alpha\left(x_{1}, x_{2}\right) \geq 1$, $\alpha\left(T x_{1}, T x_{2}\right)=\alpha\left(x_{2}, x_{3}\right) \geq 1$. Hence, by induction, we get $\alpha\left(x_{n}, x_{n+1}\right) \geq 1$ for all $n \geq 0$.

Similarly, $\beta\left(x_{n}, x_{n+1}\right) \geq 1$ for all $n \geq 0$.

Consider (2.1), we have

$$
\begin{aligned}
\psi\left(d\left(x_{n+1}, x_{n+2}\right)\right) & =\psi\left(d\left(T x_{n}, T x_{n+1}\right)\right) \\
& \leq \alpha\left(x_{n}, T x_{n}\right) \beta\left(x_{n+1}, T x_{n+1}\right) \psi\left(d\left(T x_{n}, T x_{n+1}\right)\right) \\
& \leq \theta\left(\psi\left(M\left(x_{n}, x_{n+1}\right)\right)\right) \psi\left(M\left(x_{n}, x_{n+1}\right)\right),
\end{aligned}
$$

where

$$
\begin{aligned}
M\left(x_{n}, x_{n+1}\right)= & \max \left\{d\left(x_{n}, x_{n+1}\right), d\left(x_{n}, T x_{n}\right), d\left(x_{n+1}, T x_{n+1}\right),\right. \\
& \left.\frac{d\left(x_{n}, T x_{n}\right) d\left(x_{n+1}, T x_{n+1}\right)}{1+d\left(x_{n}, x_{n+1}\right)}, \frac{d\left(x_{n}, T x_{n}\right) d\left(x_{n+1}, T x_{n+1}\right)}{1+d\left(T x_{n}, T x_{n+1}\right)}\right\} \\
= & \max \left\{d\left(x_{n}, x_{n+1}\right), d\left(x_{n}, x_{n+1}\right), d\left(x_{n+1}, x_{n+2}\right),\right. \\
& \left.\frac{d\left(x_{n}, x_{n+1}\right) d\left(x_{n+1}, x_{n+2}\right)}{1+d\left(x_{n}, x_{n+1}\right)}, \frac{d\left(x_{n}, x_{n+1}\right) d\left(x_{n+1}, x_{n+2}\right)}{1+d\left(x_{n+1}, x_{n+2}\right)}\right\} \\
= & \max \left\{d\left(x_{n}, x_{n+1}\right), d\left(x_{n+1}, x_{n+2}\right)\right\} .
\end{aligned}
$$

Now, if $M\left(x_{n}, x_{n+1}\right)=d\left(x_{n+1}, x_{n+2}\right)$, then

$$
\begin{aligned}
\psi\left(d\left(x_{n+1}, x_{n+2}\right)\right) & \leq \theta\left(\psi\left(M\left(x_{n}, x_{n+1}\right)\right)\right) \psi\left(M\left(x_{n}, x_{n+1}\right)\right) \\
& \leq \theta\left(\psi\left(M\left(x_{n}, x_{n+1}\right)\right)\right) \psi\left(d\left(x_{n+1}, x_{n+2}\right)\right) \\
& <\psi\left(d\left(x_{n+1}, x_{n+2}\right)\right),
\end{aligned}
$$

which is a contradiction, using the properties of $\psi$.

Therefore, it implies that $M\left(x_{n}, x_{n+1}\right)=d\left(x_{n}, x_{n+1}\right)$ and

$$
\begin{aligned}
\psi\left(d\left(x_{n+1}, x_{n+2}\right)\right) & \leq \theta\left(\psi\left(M\left(x_{n}, x_{n+1}\right)\right)\right) \psi\left(M\left(x_{n}, x_{n+1}\right)\right) \\
& \leq \theta\left(\psi\left(M\left(x_{n}, x_{n+1}\right)\right)\right) \psi\left(d\left(x_{n}, x_{n+1}\right)\right) \\
& \leq \psi\left(d\left(x_{n}, x_{n+1}\right)\right) .
\end{aligned}
$$


Hence, using the properties of $\psi$, we conclude that

$d\left(x_{n+1}, x_{n+2}\right) \leq d\left(x_{n}, x_{n+1}\right)$,

for every $n \in \mathbb{N}$. Therefore, sequence $\left\{d\left(x_{n}, x_{n+1}\right)\right\}$ is decreasing and for the non-negative decreasing sequence $\left\{d\left(x_{n}, x_{n+1}\right)\right\}$, there exists some $r \geq 0$, such that

$\lim _{n \rightarrow \infty} d\left(x_{n}, x_{n+1}\right)=r$.

Further from (2.3), it implies that

$\frac{\psi\left(d\left(x_{n+1}, x_{n+2}\right)\right)}{\psi\left(M\left(x_{n}, x_{n+1}\right)\right)} \leq \theta\left(\psi\left(M\left(x_{n}, x_{n+1}\right)\right)\right)<1$.

On letting $n \rightarrow \infty$ in above inequality, we have $\lim _{n \rightarrow \infty} \theta\left(\psi\left(M\left(x_{n}, x_{n+1}\right)\right)\right)=1, \quad$ and $\quad \theta \in \Theta$, $\lim _{n \rightarrow \infty} \psi\left(M\left(x_{n}, x_{n+1}\right)\right)=0$, which yields that

$r=\lim _{n \rightarrow \infty} d\left(x_{n}, x_{n+1}\right)=0$.

Now, we will show that $\left\{x_{n}\right\}$ is a Cauchy sequence. Suppose, to the contrary, that $\left\{x_{n}\right\}$ is not a Cauchy sequence.

Then, there exists $\delta>0$ for which we can find subsequences $\left\{x_{n_{k}}\right\}$ and $\left\{x_{m_{k}}\right\}$ of $\left\{x_{n}\right\}$ with $n_{k}>m_{k}>k$ such that

$d\left(x_{n_{k}}, x_{m_{k}}\right) \geq \delta$

Further, corresponding to $m_{k}$, we can choose $n_{k}$ in such a way that it is the smallest integer with $n_{k}>m_{k}$ and satisfying (2.8), we have

$d\left(x_{n_{k}-1}, x_{m_{k}}\right)<\delta$.

Using triangle inequality, we have

$0<\delta=d\left(x_{n_{k}}, x_{m_{k}}\right) \leq d\left(x_{n_{k}}, x_{n_{k}-1}\right)+d\left(x_{n_{k}-1}, x_{m_{k}}\right)<\delta$

$$
+d\left(x_{n_{k}}, x_{n_{k}-1}\right)
$$

Letting $k \rightarrow \infty$ and using (2.7) and (2.8), we obtain

$\lim _{k \rightarrow \infty} d\left(x_{n_{k}}, x_{m_{k}}\right)=\delta$

Again, using triangle inequality, we have

$d\left(x_{n_{k}}, x_{m_{k}}\right) \leq d\left(x_{n_{k}}, x_{n_{k}-1}\right)+d\left(x_{n_{k}-1}, x_{m_{k}-1}\right)+d\left(x_{m_{k}-1}, x_{m_{k}}\right)$,

and

$d\left(x_{n_{k}-1}, x_{m_{k}-1}\right) \leq d\left(x_{n_{k}}, x_{n_{k}-1}\right)+d\left(x_{n_{k}}, x_{m_{k}}\right)+d\left(x_{m_{k}-1}, x_{m_{k}}\right)$.

Therefore,

$$
\begin{array}{r}
d\left(x_{n_{k}}, x_{m_{k}}\right) \leq d\left(x_{n_{k}}, x_{n_{k}-1}\right)+d\left(x_{n_{k}-1}, x_{m_{k}-1}\right)+d\left(x_{m_{k}-1}, x_{m_{k}}\right) \\
\leq 2 d\left(x_{n_{k}}, x_{n_{k}-1}\right)+d\left(x_{n_{k}}, x_{m_{k}}\right)+2 d\left(x_{m_{k}-1}, x_{m_{k}}\right)
\end{array}
$$

Letting $k \rightarrow \infty$ in (2.12) and using (2.7), (2.11), we get

$\lim _{k \rightarrow \infty} d\left(x_{n_{k}-1}, x_{m_{k}-1}\right)=\delta$.
Put $x=x_{m_{k}}$ and $y=x_{n_{k}}$ in (2.1), we obtain

$$
\begin{aligned}
\psi\left(d\left(T x_{m_{k}}, T x_{n_{k}}\right)\right) & \leq \alpha\left(x_{m_{k}}, T x_{m_{k}}\right) \beta\left(x_{n_{k}}, T x_{n_{k}}\right) \psi\left(d\left(T x_{m_{k}}, T x_{n_{k}}\right)\right) \\
& \leq \theta\left(\psi\left(M\left(x_{m_{k}}, x_{n_{k}}\right)\right)\right) \psi\left(M\left(x_{m_{k}}, x_{n_{k}}\right)\right),
\end{aligned}
$$

where

$$
\begin{aligned}
M\left(x_{m_{k}}, x_{n_{k}}\right)= & \max \left\{d\left(x_{m_{k}}, x_{n_{k}}\right), d\left(x_{m_{k}}, T x_{m_{k}}\right), d\left(x_{n_{k}}, T x_{n_{k}}\right),\right. \\
& \left.\frac{d\left(x_{m_{k}}, T x_{m_{k}}\right) d\left(x_{n_{k}}, T x_{n_{k}}\right)}{1+d\left(x_{m_{k}}, x_{n_{k}}\right)}, \frac{d\left(x_{m_{k}}, T x_{m_{k}}\right) d\left(x_{n_{k}}, T x_{n_{k}}\right)}{1+d\left(T x_{m_{k}}, T x_{n_{k}}\right)}\right\} \\
= & \max \left\{d\left(x_{m_{k}}, x_{n_{k}}\right), d\left(x_{m_{k}}, x_{m_{k}+1}\right), d\left(x_{n_{k}}, x_{n_{k}+1}\right),\right. \\
& \left.\frac{d\left(x_{m_{k}}, x_{m_{k}+1}\right) d\left(x_{n_{k}}, x_{n_{k}+1}\right)}{1+d\left(x_{m_{k}}, x_{n_{k}}\right)}, \frac{d\left(x_{m_{k}}, x_{m_{k}+1}\right) d\left(x_{n_{k}}, x_{n_{k}+1}\right)}{1+d\left(x_{m_{k}+1}, x_{n_{k}+1}\right)}\right\}
\end{aligned}
$$

Therefore,

$\psi\left(d\left(x_{m_{k+1}}, x_{n_{k+1}}\right)\right) \leq \theta\left(\psi\left(M\left(x_{m_{k}}, x_{n_{k}}\right)\right)\right) \psi\left(M\left(x_{m_{k}}, x_{n_{k}}\right)\right)$. On taking limit $\quad k \rightarrow \infty$, we have $\psi(\delta) \leq \lim _{k \rightarrow \infty} \theta\left(\psi\left(M\left(x_{m_{k}}, x_{n_{k}}\right)\right)\right) \psi(\delta), \quad$ that is $1 \leq \lim _{k \rightarrow \infty} \theta\left(\psi\left(M\left(x_{m_{k}}, x_{n_{k}}\right)\right)\right)$, which implies that $\lim _{k \rightarrow \infty} \theta\left(\psi\left(M\left(x_{m_{k}}, x_{n_{k}}\right)\right)\right)=1$. Consequently, we obtain $\lim _{k \rightarrow \infty} M\left(x_{m_{k}}, x_{n_{k}}\right)=0$ and hence $\lim _{k \rightarrow \infty} d\left(x_{m_{k}+1}, x_{n_{k}+1}\right)=0$ which is a contradiction.

This shows that $\left\{x_{n}\right\}$ is a Cauchy sequence. Since $X$ is complete, there exists $x^{*} \in X$ such that $x_{n} \rightarrow x^{*}$.

First, we suppose that $T$ is continuous. Therefore, we have

$x^{*}=\lim _{n \rightarrow \infty} x_{n+1}=\lim _{n \rightarrow \infty} T x_{n}=T \lim _{n \rightarrow \infty} x_{n}=T x^{*}$.

Now, we suppose that $X$ is $(\alpha, \beta)$-regular.

Therefore, there exists a subsequence $\left\{x_{n_{k}}\right\}$ of $\left\{x_{n}\right\}$ such that $\alpha\left(x_{n_{k}-1}, x_{n_{k}}\right) \geq 1$ and $\beta\left(x_{n_{k}-1}, x_{n_{k}}\right) \geq 1$ for all $k \in \mathbb{N}$ and $\alpha\left(x^{*}, T x^{*}\right) \geq 1$ and $\beta\left(x^{*}, T x^{*}\right) \geq 1$. Now, from inequality (2.1) with $x=x_{n_{k}}$ and $y=x^{*}$, we obtain

$$
\begin{aligned}
\psi\left(d\left(x_{n_{k}+1}, T x^{*}\right)\right)= & \psi\left(d\left(T x_{n_{k}}, T x^{*}\right)\right) \\
& \left.\leq \alpha\left(x_{n_{k}}, T x_{n_{k}}\right) \beta\left(x^{*}, T x^{*}\right) \psi\left(d\left(T x_{n_{k}}, T x^{*}\right)\right)\right) \\
& \leq \theta\left(\psi\left(M\left(x_{n_{k}}, x^{*}\right)\right)\right) \psi\left(M\left(x_{n_{k}}, x^{*}\right)\right),
\end{aligned}
$$

where

$$
\begin{aligned}
M\left(x_{n_{k}}, x^{*}\right)= & \max \left\{d\left(x_{n_{k}}, x^{*}\right), d\left(x_{n_{k}}, T x_{n_{k}}\right), d\left(x^{*}, T x^{*}\right),\right. \\
& \left.\frac{d\left(x_{n_{k}}, T x_{n_{k}}\right) d\left(x^{*}, T x^{*}\right)}{1+d\left(x_{n_{k}}, x^{*}\right)}, \frac{d\left(x_{n_{k}}, T x_{n_{k}}\right) d\left(x^{*}, T x^{*}\right)}{1+d\left(T x_{n_{k}}, T x^{*}\right)}\right\} \\
= & \max \left\{d\left(x_{n_{k}}, x^{*}\right), d\left(x_{n_{k}}, x_{n_{k}+1}\right), d\left(x^{*}, T x^{*}\right), .\right. \\
& \left.\frac{d\left(x_{n_{k}}, x_{n_{k}+1}\right) d\left(x^{*}, T x^{*}\right)}{1+d\left(x_{n_{k}}, x^{*}\right)}, \frac{d\left(x_{n_{k}}, x_{n_{k}+1}\right) d\left(x^{*}, T x^{*}\right)}{1+d\left(x_{n_{k}+1}, T x^{*}\right)}\right\} .
\end{aligned}
$$


Therefore,

$\psi\left(d\left(x_{n_{k}+1}, T x^{*}\right)\right) \leq \theta\left(\psi\left(M\left(x_{n_{k}}, x^{*}\right)\right)\right) \psi\left(M\left(x_{n_{k}}, x^{*}\right)\right)$. On taking limit $k \rightarrow \infty$, we have $\psi\left(d\left(x^{*}, T x^{*}\right)\right) \leq \lim _{k \rightarrow \infty}$ $\theta\left(\psi\left(M\left(x_{n_{k}}, x^{*}\right)\right)\right) \psi\left(d\left(x^{*}, T x^{*}\right)\right)$, that is $1 \leq \lim _{k \rightarrow \infty} \theta(\psi(M$ $\left.\left.\left(x_{n_{k}}, x^{*}\right)\right)\right)$, which implies that $\lim _{k \rightarrow \infty} \theta\left(\psi\left(M\left(x_{n_{k}}, x^{*}\right)\right)\right)$ $=1$. Consequently, we obtain $\lim _{k \rightarrow \infty} M\left(x_{n_{k}}, x^{*}\right)=0$ and hence $d\left(x^{*}, T x^{*}\right)=0$, that is, $x^{*}=T x^{*}$.

Further, suppose that $x^{*}$ and $y^{*}$ are two fixed points of $T$ such that $x^{*} \neq y^{*}$ and $\alpha\left(x^{*}, T x^{*}\right) \geq 1, \alpha\left(y^{*}, T y^{*}\right) \geq 1$ and $\beta\left(x^{*}, T x^{*}\right) \geq 1, \beta\left(y^{*}, T y^{*}\right) \geq 1$. Now applying (2.1), we have

$$
\begin{aligned}
\psi\left(d\left(x^{*}, y^{*}\right)\right)= & \psi\left(d\left(T x^{*}, T y^{*}\right)\right) \\
& \leq \alpha\left(x^{*}, T x^{*}\right) \beta\left(y^{*}, T y^{*}\right) \psi\left(d\left(T x^{*}, T y^{*}\right)\right) \\
& \leq \theta\left(\psi\left(M\left(x^{*}, y^{*}\right)\right)\right) \psi\left(M\left(x^{*}, y^{*}\right)\right)
\end{aligned}
$$

where

$$
\begin{aligned}
M\left(x^{*}, y^{*}\right)= & \max \left\{d\left(x^{*}, y^{*}\right), d\left(x^{*}, T x^{*}\right), d\left(y^{*}, T y^{*}\right),\right. \\
& \left.\frac{d\left(x^{*}, T x^{*}\right) d\left(y^{*}, T y^{*}\right)}{1+d\left(x^{*}, y^{*}\right)}, \frac{d\left(x^{*}, T x^{*}\right) d\left(y^{*}, T y^{*}\right)}{1+d\left(T x^{*}, T y^{*}\right)}\right\} .
\end{aligned}
$$

Hence,

$\psi\left(d\left(x^{*}, y^{*}\right)\right) \leq \theta\left(\psi\left(M\left(x^{*}, y^{*}\right)\right)\right) \psi\left(d\left(x^{*}, y^{*}\right)\right)<\psi\left(d\left(x^{*}, y^{*}\right)\right)$, which is a contradiction unless $d\left(x^{*}, y^{*}\right)=0$, that is, $x^{*}=y^{*}$. Hence, $T$ has a unique fixed point.

Example 2.1 Let $X=[0, \infty)$ be endowed with the usual metric $d(x, y)=|x-y|$ for all $x, y \in X$ and $T: X \rightarrow X$ be defined by

$$
T x=\left\{\begin{array}{cl}
\frac{1-x^{2}}{8}, & x \in[0,1] \\
9 x, & x \in(1, \infty)
\end{array}\right.
$$

Define also $\alpha, \beta: X \times X \rightarrow \mathbb{R}^{+}, \theta:[0, \infty) \rightarrow[0,1)$ and $\psi:$ $[0, \infty) \rightarrow[0, \infty)$ as

$\alpha(x, y)= \begin{cases}1, & (x, y) \in[0,1] \\ 0, & \text { otherwise }\end{cases}$

$\beta(x, y)= \begin{cases}1, & (x, y) \in[0,1] \\ 0, & \text { otherwise }\end{cases}$

$\theta(t)=\frac{1}{2}$ and $\psi(t)=t$.

Now, we prove that Theorem 2.1 can be applied to $T$ (here, a fixed point is $u=\sqrt{17}-4$ ), but Theorem 1.1 cannot be applied to $T$.

Clearly, $(X, d)$ is a complete metric space. We show that $T$ is an $(\alpha, \beta)$-admissible mapping. Let $x, y \in X$, if $\alpha(x, y) \geq 1$ and $\beta(x, y) \geq 1$, then $x, y \in[0,1]$. On the other hand, for all $x \in[0,1]$, we have $T x \leq 1$. It follows that $\alpha(T x, T y) \geq 1$ and $\beta(T x, T y) \geq 1$. Thus, the assertion holds. In reason of the above arguments, $\alpha(0, T 0) \geq 1$ and $\beta(0, T 0) \geq 1$.
Now, if $\left\{x_{n}\right\}$ is a sequence in $X$ such that $\alpha\left(x_{n}, x_{n+1}\right) \geq 1, \beta\left(x_{n}, x_{n+1}\right) \geq 1$ and $x_{n} \rightarrow x \in X$, for all $n \in \mathbb{N} \cup\{0\}$, then $x_{n} \subseteq[0,1]$ and hence $x \in[0,1]$. This implies that $\alpha(x, T x) \geq 1$, and $\beta(x, T x) \geq 1$.

Let $x, y \in[0,1]$. We get

$$
\begin{aligned}
\alpha(x, T x) \beta(y, T y) \psi(d(T x, T y))= & |T x-T y|=\frac{1}{8}|x-y| \mid \\
& \times x+y\left|\leq \frac{1}{2}\right| x-y \mid \\
= & \theta(\psi(d(x, y))) \psi(d(x, y)) .
\end{aligned}
$$

Hence, the given inequality is satisfied.

Otherwise, if $\alpha(x, T x) \beta(y, T y)=0 . \quad$ Then, $\alpha(x, T x)$ $\beta(y, T y) \psi(d(T x, T y))=0 \leq \theta(\psi(M(x, y))) \psi(M(x, y))$.

Therefore, all the conditions of Theorem 2.1 are satisfied and $T$ has a fixed point.

Now, let $x=3, y=4$. We get

$d(T 3, T 4)=9>\frac{1}{2}=\frac{1}{2}|3-4|=\theta(d(3,4)) d(3,4)$.

Therefore, Theorem 1.1 does not hold for this example.

Definition 2.2 Let $(X, d)$ be a metric space, $T: X \rightarrow X$ and $\alpha, \beta: X \times X \rightarrow \mathbb{R}^{+}$. A mapping $T$ is said to be $\alpha, \beta$-Geraghty type-II rational contractive mapping if there exists a $\theta \in \Theta$, such that for all $x, y \in X$, following condition holds:

$[\psi(d(T x, T y))+l]^{\alpha(x, T x) \beta(y, T y)} \leq \theta(\psi(M(x, y))) \psi(M(x, y))+l$,

where $\quad M(x, y)=\max \{d(x, y), d(x, T x), d(y, T y), \underline{d(x, T x)}$ $\left.d(y, T y) 1+d(x, y), \frac{d(x, T x) d(y, T y)}{1+d(T x, T y)}\right\}, \psi \in \Psi$ and $l \geq 1$.

Theorem 2.2 Let $(X, d)$ be a complete metric space, $T$ is self-mapping, $T: X \rightarrow X$, and $\alpha, \beta: X \times X \rightarrow \mathbb{R}$. Suppose that the following conditions are satisfied:

(i) $T$ is an $(\alpha, \beta)$-admissible mapping;

(ii) $T$ is an $(\alpha, \beta)$-Geraghty type-II rational contractive mapping;

(iii) there exists $x_{0} \in X$ such that $\alpha\left(x_{0}, T x_{0}\right) \geq 1$ and $\beta\left(x_{0}, T x_{0}\right) \geq 1$;

(iv) either $T$ is continuous or $X$ is $(\alpha, \beta)$-regular.

Then, Thas a fixed point $x^{*} \in X$ and $\left\{T^{n} x_{0}\right\}$ converges to $x^{*}$.

Further, if for all $x, y \in F(T)$, with $x \neq y$ such that $\alpha(x, T x) \geq 1, \alpha(y, T y) \geq 1$ and $\beta(x, T x) \geq 1, \quad \beta(y, T y) \geq 1$, then $T$ has a unique fixed point in $X$.

Proof Let $x_{0} \in X$ such that $\alpha\left(x_{0}, T x_{0}\right) \geq 1$ and $\beta\left(x_{0}, T x_{0}\right) \geq 1$. Now, we can construct the sequences $\left\{x_{n}\right\}$ in $X$ by $x_{n}=T^{n} x_{0}=T x_{n-1}$, for $n \in \mathbb{N}$.

Moreover, we assume that if $x_{n_{0}}=x_{n_{0}+1}$, for some $n_{0} \in \mathbb{N}$, then $x_{n_{0}}$ is a fixed point of $T$. Consequently, we suppose that $x_{n} \neq x_{n+1}$ for all $n \in \mathbb{N}$. 
Since $T$ is $(\alpha, \beta)$-admissible mapping, $\alpha\left(x_{0}, T x_{0}\right)=$ $\alpha\left(x_{0}, x_{1}\right) \geq 1, \quad \alpha\left(T x_{0}, T x_{1}\right)=\alpha\left(x_{1}, x_{2}\right) \geq 1, \quad \alpha\left(T x_{1}, T x_{2}\right)=$ $\alpha\left(x_{2}, x_{3}\right) \geq 1$. Hence, by induction, we get $\alpha\left(x_{n}, x_{n+1}\right) \geq 1$ for all $n \geq 0$.

Similarly, $\beta\left(x_{n}, x_{n+1}\right) \geq 1$ for all $n \geq 0$.

Consider (2.19), we have

$$
\begin{aligned}
\psi\left(d\left(x_{n+1}, x_{n+2}\right)\right)+l & =\psi\left(d\left(T x_{n}, T x_{n+1}\right)\right)+l \\
& \leq\left[\psi\left(d\left(T x_{n}, T x_{n+1}\right)\right)+l\right]^{\alpha\left(x_{n}, T x_{n}\right) \beta\left(x_{n+1}, T x_{n+1}\right)} \\
& \leq \theta\left(\psi\left(M\left(x_{n}, x_{n+1}\right)\right)\right) \psi\left(M\left(x_{n}, x_{n+1}\right)\right)+l,
\end{aligned}
$$

where

$$
\begin{aligned}
M\left(x_{n}, x_{n+1}\right)= & \max \left\{d\left(x_{n}, x_{n+1}\right), d\left(x_{n}, T x_{n}\right), d\left(x_{n+1}, T x_{n+1}\right),\right. \\
& \left.\frac{d\left(x_{n}, T x_{n}\right) d\left(x_{n+1}, T x_{n+1}\right)}{1+d\left(x_{n}, x_{n+1}\right)}, \frac{d\left(x_{n}, T x_{n}\right) d\left(x_{n+1}, T x_{n+1}\right)}{1+d\left(T x_{n}, T x_{n+1}\right)}\right\} \\
= & \max \left\{d\left(x_{n}, x_{n+1}\right), d\left(x_{n}, x_{n+1}\right), d\left(x_{n+1}, x_{n+2}\right),\right. \\
& \left.\frac{d\left(x_{n}, x_{n+1}\right) d\left(x_{n+1}, x_{n+2}\right)}{1+d\left(x_{n}, x_{n+1}\right)}, \frac{d\left(x_{n}, x_{n+1}\right) d\left(x_{n+1}, x_{n+2}\right)}{1+d\left(x_{n+1}, x_{n+2}\right)}\right\} \\
= & \max \left\{d\left(x_{n}, x_{n+1}\right), d\left(x_{n+1}, x_{n+2}\right)\right\} .
\end{aligned}
$$

Now, if $M\left(x_{n}, x_{n+1}\right)=d\left(x_{n+1}, x_{n+2}\right)$, then

$$
\begin{aligned}
\psi\left(d\left(x_{n+1}, x_{n+2}\right)\right) & \leq \theta\left(\psi\left(M\left(x_{n}, x_{n+1}\right)\right)\right) \psi\left(M\left(x_{n}, x_{n+1}\right)\right) \\
& \leq \theta\left(\psi\left(M\left(x_{n}, x_{n+1}\right)\right)\right) \psi\left(d\left(x_{n+1}, x_{n+2}\right)\right) \\
& <\psi\left(d\left(x_{n+1}, x_{n+2}\right)\right),
\end{aligned}
$$

which is a contradiction, using the properties of $\psi$.

Therefore, it implies that $M\left(x_{n}, x_{n+1}\right)=d\left(x_{n}, x_{n+1}\right)$ and

$$
\begin{aligned}
\psi\left(d\left(x_{n+1}, x_{n+2}\right)\right) & \leq \theta\left(\psi\left(M\left(x_{n}, x_{n+1}\right)\right)\right) \psi\left(M\left(x_{n}, x_{n+1}\right)\right) \\
& \leq \theta\left(\psi\left(M\left(x_{n}, x_{n+1}\right)\right)\right) \psi\left(d\left(x_{n}, x_{n+1}\right)\right) \\
& \leq \psi\left(d\left(x_{n}, x_{n+1}\right)\right) .
\end{aligned}
$$

Hence, using the properties of $\psi$, we conclude that

$d\left(x_{n+1}, x_{n+2}\right) \leq d\left(x_{n}, x_{n+1}\right)$,

for every $n \in \mathbb{N}$. Therefore, sequence $\left\{d\left(x_{n}, x_{n+1}\right)\right\}$ is decreasing. On the similar lines as in Theorem 2.1, we can prove that

$r=\lim _{n \rightarrow \infty} d\left(x_{n}, x_{n+1}\right)=0$.

Now, we will show that $\left\{x_{n}\right\}$ is a Cauchy sequence. Suppose, to the contrary, that $\left\{x_{n}\right\}$ is not a Cauchy sequence.

Then, there exists $\delta>0$ for which we can find subsequences $\left\{x_{n_{k}}\right\}$ and $\left\{x_{m_{k}}\right\}$ of $\left\{x_{n}\right\}$ with $n_{k}>m_{k}>k$ such that

$d\left(x_{n_{k}}, x_{m_{k}}\right) \geq \delta$.
Further, corresponding to $m_{k}$, we can choose $n_{k}$ in such a way that it is the smallest integer with $n_{k}>m_{k}$ and satisfying (2.24), we have

$d\left(x_{n_{k}-1}, x_{m_{k}}\right)<\delta$.

Using triangle inequality, we have

$$
\begin{aligned}
0<\delta= & d\left(x_{n_{k}}, x_{m_{k}}\right) \leq d\left(x_{n_{k}}, x_{n_{k}-1}\right)+d\left(x_{n_{k}-1}, x_{m_{k}}\right)<\delta \\
& +d\left(x_{n_{k}}, x_{n_{k}-1}\right)
\end{aligned}
$$

Letting $k \rightarrow \infty$ and using (2.23) and (2.24), we obtain

$\lim _{k \rightarrow \infty} d\left(x_{n_{k}}, x_{m_{k}}\right)=\delta$.

Again, using triangle inequality, we have

$d\left(x_{n_{k}}, x_{m_{k}}\right) \leq d\left(x_{n_{k}}, x_{n_{k}-1}\right)+d\left(x_{n_{k}-1}, x_{m_{k}-1}\right)+d\left(x_{m_{k}-1}, x_{m_{k}}\right)$,

and

$d\left(x_{n_{k}-1}, x_{m_{k}-1}\right) \leq d\left(x_{n_{k}}, x_{n_{k}-1}\right)+d\left(x_{n_{k}}, x_{m_{k}}\right)+d\left(x_{m_{k}-1}, x_{m_{k}}\right)$.

Therefore,

$$
\begin{gathered}
d\left(x_{n_{k}}, x_{m_{k}}\right) \leq d\left(x_{n_{k}}, x_{n_{k}-1}\right)+d\left(x_{n_{k}-1}, x_{m_{k}-1}\right)+d\left(x_{m_{k}-1}, x_{m_{k}}\right) \\
\leq 2 d\left(x_{n_{k}}, x_{n_{k}-1}\right)+d\left(x_{n_{k}}, x_{m_{k}}\right)+2 d\left(x_{m_{k}-1}, x_{m_{k}}\right)
\end{gathered}
$$

Letting $k \rightarrow \infty$ in (2.28) and using (2.23), (2.27), we get

$\lim _{k \rightarrow \infty} d\left(x_{n_{k}-1}, x_{m_{k}-1}\right)=\delta$.

Put $x=x_{m_{k}}$ and $y=x_{n_{k}}$ in (2.19), we obtain

$$
\begin{aligned}
\psi\left(d\left(T x_{m_{k}}, T x_{n_{k}}\right)\right)+l & \leq\left[\psi\left(d\left(T x_{m_{k}}, T x_{n_{k}}\right)\right)+l\right]^{\alpha\left(x_{m_{k}}, T x_{m_{k}}\right) \beta\left(x_{n_{k}}, T x_{n_{k}}\right)} \\
& \leq \theta\left(\psi\left(M\left(x_{m_{k}}, x_{n_{k}}\right)\right)\right) \psi\left(M\left(x_{m_{k}}, x_{n_{k}}\right)\right)+l,
\end{aligned}
$$

where

$$
\begin{aligned}
M\left(x_{m_{k}}, x_{n_{k}}\right)= & \max \left\{d\left(x_{m_{k}}, x_{n_{k}}\right), d\left(x_{m_{k}}, T x_{m_{k}}\right), d\left(x_{n_{k}}, T x_{n_{k}}\right),\right. \\
& \left.\frac{d\left(x_{m_{k}}, T x_{m_{k}}\right) d\left(x_{n_{k}}, T x_{n_{k}}\right)}{1+d\left(x_{m_{k}}, x_{n_{k}}\right)}, \frac{d\left(x_{m_{k}}, T x_{m_{k}}\right) d\left(x_{n_{k}}, T x_{n_{k}}\right)}{1+d\left(T x_{m_{k}}, T x_{n_{k}}\right)}\right\} \\
= & \max \left\{d\left(x_{m_{k}}, x_{n_{k}}\right), d\left(x_{m_{k}}, x_{m_{k}+1}\right), d\left(x_{n_{k}}, x_{n_{k}+1}\right),\right. \\
& \left.\frac{d\left(x_{m_{k}}, x_{m_{k}+1}\right) d\left(x_{n_{k}}, x_{n_{k}+1}\right)}{1+d\left(x_{m_{k}}, x_{n_{k}}\right)}, \frac{d\left(x_{m_{k}}, x_{m_{k}+1}\right) d\left(x_{n_{k}}, x_{n_{k}+1}\right)}{1+d\left(x_{m_{k}+1}, x_{n_{k}+1}\right)}\right\} .
\end{aligned}
$$

Therefore, $\quad \psi\left(d\left(x_{m_{k+1}}, x_{n_{k+1}}\right)\right) \leq \theta\left(\psi\left(M\left(x_{m_{k}}, x_{n_{k}}\right)\right)\right) \psi$ $\left(M\left(x_{m_{k}}, x_{n_{k}}\right)\right)$. On taking limit $k \rightarrow \infty$, we have $\psi(\delta) \leq \lim _{k \rightarrow \infty} \theta\left(\psi\left(M\left(x_{m_{k}}, x_{n_{k}}\right)\right)\right) \psi(\delta)$, that is $1 \leq \lim _{k \rightarrow \infty}$ $\theta\left(\psi\left(M\left(x_{m_{k}}, x_{n_{k}}\right)\right)\right)$, which implies that $\lim _{k \rightarrow \infty} \theta$ $\left(\psi\left(M\left(x_{m_{k}}, x_{n_{k}}\right)\right)\right)=1$. Consequently, we obtain $\lim _{k \rightarrow \infty}$ $M\left(x_{m_{k}}, x_{n_{k}}\right)=0$ and hence $\lim _{k \rightarrow \infty} d\left(x_{m_{k}+1}, x_{n_{k}+1}\right)=0$ which is a contradiction. 
This shows that $\left\{x_{n}\right\}$ is a Cauchy sequence. Since $X$ is complete, there exists $x^{*} \in X$ such that $x_{n} \rightarrow x^{*}$.

First, we suppose that $T$ is continuous. Therefore, we have

$x^{*}=\lim _{n \rightarrow \infty} x_{n+1}=\lim _{n \rightarrow \infty} T x_{n}=T \lim _{n \rightarrow \infty} x_{n}=T x^{*}$.

Now, we suppose that $X$ is $\alpha, \beta$-regular.

Therefore, there exists a subsequence $\left\{x_{n_{k}}\right\}$ of $\left\{x_{n}\right\}$ such that $\alpha\left(x_{n_{k}-1}, x_{n_{k}}\right) \geq 1$ and $\beta\left(x_{n_{k}-1}, x_{n_{k}}\right) \geq 1$ for all $k \in \mathbb{N}$ and $\alpha\left(x^{*}, T x^{*}\right) \geq 1$ and $\beta\left(x^{*}, T x^{*}\right) \geq 1$. Now, from inequality (2.19) with $x=x_{n_{k}}$ and $y=x^{*}$, we obtain

$$
\begin{aligned}
\psi\left(d\left(x_{n_{k}+1}, T x^{*}\right)\right)+l & =\psi\left(d\left(T x_{n_{k}}, T x^{*}\right)\right)+l \\
& \left.\leq\left[\psi\left(d\left(T x_{n_{k}}, T x^{*}\right)\right)\right)+l\right]^{\alpha\left(x_{n_{k}}, T x_{n_{k}}\right) \beta\left(x^{*}, T x^{*}\right)} \\
& \leq \theta\left(\psi\left(M\left(x_{n_{k}}, x^{*}\right)\right)\right) \psi\left(M\left(x_{n_{k}}, x^{*}\right)\right)+l,
\end{aligned}
$$

where

$$
\begin{aligned}
M\left(x_{n_{k}}, x^{*}\right)= & \max \left\{d\left(x_{n_{k}}, x^{*}\right), d\left(x_{n_{k}}, T x_{n_{k}}\right), d\left(x^{*}, T x^{*}\right),\right. \\
& \left.\frac{d\left(x_{n_{k}}, T x_{n_{k}}\right) d\left(x^{*}, T x^{*}\right)}{1+d\left(x_{n_{k}}, x^{*}\right)}, \frac{d\left(x_{n_{k}}, T x_{n_{k}}\right) d\left(x^{*}, T x^{*}\right)}{1+d\left(T x_{n_{k}}, T x^{*}\right)}\right\} \\
= & \max \left\{d\left(x_{n_{k}}, x^{*}\right), d\left(x_{n_{k}}, x_{n_{k}+1}\right), d\left(x^{*}, T x^{*}\right),\right. \\
& \left.\frac{d\left(x_{n_{k}}, x_{n_{k}+1}\right) d\left(x^{*}, T x^{*}\right)}{1+d\left(x_{n_{k}}, x^{*}\right)}, \frac{d\left(x_{n_{k}}, x_{n_{k}+1}\right) d\left(x^{*}, T x^{*}\right)}{1+d\left(x_{n_{k}+1}, T x^{*}\right)}\right\} .
\end{aligned}
$$

Therefore,

$\psi\left(d\left(x_{n_{k}+1}, T x^{*}\right)\right) \leq \theta\left(\psi\left(M\left(x_{n_{k}}, x^{*}\right)\right)\right) \psi\left(M\left(x_{n_{k}}, x^{*}\right)\right)$. On taking limit $k \rightarrow \infty$, we have $\psi\left(d\left(x^{*}, T x^{*}\right)\right) \leq \lim _{k \rightarrow \infty} \theta\left(\psi\left(M\left(x_{n_{k}}, x^{*}\right)\right)\right) \psi\left(d\left(x^{*}, T x^{*}\right)\right)$,

that is $1 \leq \lim _{k \rightarrow \infty} \theta\left(\psi\left(M\left(x_{n_{k}}, x^{*}\right)\right)\right)$, which implies that $\lim _{k \rightarrow \infty} \theta\left(\psi\left(M\left(x_{n_{k}}, x^{*}\right)\right)\right)=1$. Consequently, we obtain $\lim _{k \rightarrow \infty} M\left(x_{n_{k}}, x^{*}\right)=0$ and hence $d\left(x^{*}, T x^{*}\right)=0$, that is, $x^{*}=T x^{*}$.

Further, suppose that $x^{*}$ and $y^{*}$ are two fixed points of $T$ such that $x^{*} \neq y^{*}$ and $\alpha\left(x^{*}, T x^{*}\right) \geq 1, \alpha\left(y^{*}, T y^{*}\right) \geq 1$ and $\beta\left(x^{*}, T x^{*}\right) \geq 1, \beta\left(y^{*}, T y^{*}\right) \geq 1$. Hence from (2.19), we have

$$
\begin{aligned}
\psi\left(d\left(x^{*}, y^{*}\right)\right)+l & =\psi\left(d\left(T x^{*}, T y^{*}\right)\right)+l \\
& \leq\left[\psi\left(d\left(T x^{*}, T y^{*}\right)\right)+l\right]^{\alpha\left(x^{*}, T x^{*}\right) \beta\left(y^{*}, T y^{*}\right)} \\
& \leq \theta\left(\psi\left(M\left(x^{*}, y^{*}\right)\right)\right) \psi\left(M\left(x^{*}, y^{*}\right)\right)+l
\end{aligned}
$$

where

$$
\begin{aligned}
M\left(x^{*}, y^{*}\right)= & \max \left\{d\left(x^{*}, y^{*}\right), d\left(x^{*}, T x^{*}\right), d\left(y^{*}, T y^{*}\right),\right. \\
& \left.\frac{d\left(x^{*}, T x^{*}\right) d\left(y^{*}, T y^{*}\right)}{1+d\left(x^{*}, y^{*}\right)}, \frac{d\left(x^{*}, T x^{*}\right) d\left(y^{*}, T y^{*}\right)}{1+d\left(T x^{*}, T y^{*}\right)}\right\} .
\end{aligned}
$$

Hence, $\psi\left(d\left(x^{*}, y^{*}\right)\right) \leq \theta\left(\psi\left(M\left(x^{*}, y^{*}\right)\right)\right) \psi\left(d\left(x^{*}, y^{*}\right)\right)$, which implies that $\theta\left(\psi\left(M\left(x^{*}, y^{*}\right)\right)\right)=1$. Therefore, $d\left(x^{*}, y^{*}\right)=0$, that is, $x^{*}=y^{*}$. Hence, $T$ has a unique fixed point.

Example 2.2 Let $X=[0, \infty)$ be endowed with the usual metric $d(x, y)=|x-y|$ for all $x, y \in X$ and $T: X \rightarrow X$ be defined by

$$
T x=\left\{\begin{array}{cc}
\frac{x}{x+1}, & x \in[0,1] \\
3 x, & x \in(1, \infty)
\end{array}\right.
$$

Define also $\alpha, \beta: X \times X \rightarrow \mathbb{R}^{+}, \theta:[0, \infty) \rightarrow[0,1)$ and $\psi:$ $[0, \infty) \rightarrow[0, \infty)$ as

$$
\begin{aligned}
& \alpha(x, y)= \begin{cases}1, & (x, y) \in[0,1] \\
0, & \text { otherwise }\end{cases} \\
& \beta(x, y)= \begin{cases}1, & (x, y) \in[0,1] \\
0, & \text { otherwise }\end{cases}
\end{aligned}
$$

$\theta(t)=\frac{1}{1+t}$ and $\psi(t)=t$.

Now, we prove that Theorem 2.2 can be applied to $T$ (here, a fixed point is $u=0$ ), but Theorem 1.1 cannot be applied to $T$.

Clearly, $(X, d)$ is a complete metric space. On the same lines of Example 2.1, we can show that $T$ is an $(\alpha, \beta)$ admissible mapping.

Let $x, y \in[0,1]$. We get

$$
\begin{aligned}
{[\psi(d(T x, T y))+l]^{\alpha(x, T x) \beta(y, T y)}=} & \psi(d(T x, T y))+l \\
& =|T x-T y|+l=\left|\frac{x}{x+1}-\frac{y}{y+1}\right|+l \\
& =\left|\frac{y-x}{(1+x)(1+y)}\right|+l \\
& \leq\left|\frac{y-x}{1+y-x}\right|+l \\
& =\theta(\psi(d(x, y))) \psi(d(x, y))+l .
\end{aligned}
$$

Hence, the given inequality is satisfied.

Otherwise, $\alpha(x, T x) \beta(y, T y)=0$ and $[\psi(d(T x, T y))+$ $l]^{\alpha(x, T x) \beta(y, T y)}=1 \leq \theta(\psi(M(x, y))) \psi(M(x, y))+l$. Therefore, all the conditions of Theorem 2.2 are satisfied and $T$ has a fixed point.

Now, let $x=3, y=4$. We get

$$
d(T 3, T 4)=3>\frac{1}{2}=\frac{1}{1+|3-4|}|3-4|=\theta(d(3,4)) d(3,4) \text {. }
$$

Therefore, Theorem 1.1 does not hold for this example.

\section{Consequences of the main results}

In this section, we discuss some consequences of our main results. First, we prove some fixed point theorems for cyclic mappings in metric and ordered metric spaces. Also, 
we obtain some other related interesting results in the next section. In the last section, we obtain a fixed point theorem whenever the space is endowed with a graph.

\section{Fixed point results for cyclic mappings in metric and ordered metric spaces}

Theorem 3.1 Let $(X, d)$ be a complete metric space, $A$ and $B$ be two nonempty closed subsets of $X$. Suppose that $\alpha: X \times X \rightarrow[0, \infty)$, and $T: A \cup B \rightarrow A \cup B$ be a mapping with $T A \subseteq B$ and $T B \subseteq A$, such that $\alpha(T y, T x) \geq 1$ if $\alpha(x, y) \geq 1$, where $x \in A$ and $y \in B$. Further assume that $T$ satisfies any one of the following contractive condition for all $x \in A$ and $y \in B$

$\alpha(x, y) \psi(d(T x, T y)) \leq \theta(\psi(M(x, y))) \psi(M(x, y)) ;$

or

$[\psi(d(T x, T y))+l]^{\alpha(x, y)} \leq \theta(\psi(M(x, y))) \psi(M(x, y))+l$,

where $\quad M(x, y)=\max \{d(x, y), d(x, T x), d(y, T y), \underline{d(x, T x)}$

$\left.d(y, T y) 1+d(x, y), \frac{d(x, T x) d(y, T y)}{1+d(T x, T y)}\right\}, \psi \in \Psi$ and $l \geq 1$.

If there exists $x_{0} \in A$ such that $\alpha\left(x_{0}, T x_{0}\right) \geq 1$, and either $T$ is continuous or $X$ is $\alpha$-regular, then $T$ has a fixed point in $A \cap B$.

Proof Let $Y=A \cup B$ and $\beta: Y \times Y \rightarrow[0, \infty)$ defined as $\beta(x, y)= \begin{cases}1, & \text { if } x \in A, \quad y \in B, \\ 0, & \text { otherwise }\end{cases}$

Then, $(Y, d)$ is complete metric space. Now, if $x_{0} \in A$ is such that $\alpha\left(x_{0}, T x_{0}\right) \geq 1$, then also $\beta\left(x_{0}, T x_{0}\right) \geq 1$ and hence all the hypotheses of above Theorems 2.1-2.2 hold with $X=Y$. Consequently, $T$ has a fixed point in $A \cup B$, say z. Since $z \in A$ implies $z=T z \in B$ and $z \in B$ implies $z=T z \in A$, then $z \in A \cap B$.

Now, assume that $\left\{x_{n}\right\}$ be a sequence in $Y$ such that $\alpha\left(x_{2 n}, x_{2 n+1}\right) \geq 1$ and $\beta\left(x_{2} n, x_{2 n+1}\right) \geq 1$ for all $n \in \mathbb{N} \cup\{0\}$ and $x_{n} \rightarrow x$ as $n \rightarrow \infty$, then $x_{2 n} \in A$ and $x_{2 n+1} \in B$. Since $B$ is closed, then $x \in B$ and hence $\alpha\left(x_{2 n}, x\right) \geq 1$ and $\beta\left(x_{2 n}, x\right) \geq 1$. We deduce that all the hypotheses of above Theorems 2.1-2.2 are satisfied with $X=Y$ and hence $T$ has a fixed point.

Theorem 3.2 Let $(X, d, \leq)$ be an ordered complete metric space, $A$ and $B$ be two nonempty closed subsets of $X$. Suppose that $\alpha: X \times X \rightarrow[0, \infty)$, and $T: A \cup B \rightarrow A \cup B$ be a mapping with $T A \subseteq B$ and $T B \subseteq A$, such that $\alpha(T y, T x) \geq 1$ if $\alpha(x, y) \geq 1$, where $x \in A$ and $y \in B$. Further assume that $T$ satisfies any one of the following contractive condition for all $x \in A$ and $y \in B$ with $x \leq y$

$\alpha(x, y) \psi(d(T x, T y)) \leq \theta(\psi(M(x, y))) \psi(M(x, y)) ;$

or
$[\psi(d(T x, T y))+l]^{\alpha(x, y)} \leq \theta(\psi(M(x, y))) \psi(M(x, y))+l$,

where $M(x, y)=\max \{d(x, y), d(x, T x), d(y, T y), \underline{d(x, T x) d(y, T y)}$ $\left.1+d(x, y), \frac{d(x, T x) d(y, T y)}{1+d(T x, T y)}\right\}, \psi \in \Psi$ and $l \geq 1$.

If there exists $x_{0} \in A$ such that $\alpha\left(x_{0}, T x_{0}\right) \geq 1$ and $x_{0} \leq T x_{0}$, and either $T$ is continuous and decreasing or $X$ is an ordered $\alpha$-regular, then $T$ has a fixed point in $A \cup B$.

Proof Consider the complete metric space $(Y, d)$, where $Y=A \cup B$ and define $\beta: Y \times Y \rightarrow[0, \infty)$ defined as

$\beta(x, y)= \begin{cases}1, & \text { if } x \in A, \quad y \in B, \text { with } x \leq y \\ 0, & \text { otherwise }\end{cases}$

Let $\beta(x, y) \geq 1$ for $x, y \in X$, then $x \in A$ and $y \in B$ with $x \leq y$. It follows that $T x \in B$ and $T y \in A$ with $T y \leq T x$, since $T$ is decreasing. Therefore, $\beta(T y, T x) \geq 1$, that is, $T$ is an $(\alpha$, $\beta$ )-admissible mapping. Now, let $\alpha\left(x_{0}, T x_{0}\right) \geq 1$ with $x_{0} \in$ $A$ and $x_{0} \leq T x_{0}$. From $x_{0} \in A$ we have $T x_{0} \in B$ with $x_{0} \leq T x_{0}$, that is, $\beta\left(x_{0}, T x_{0}\right) \geq 1$. Hence, all the hypotheses of above Theorems 2.1-2.2 hold with $X=Y$. Consequently, $T$ has a fixed point in $A \cup B$, say $z$. Since $z \in A$ implies $z=T z \in B$ and $z \in B$ implies $z=T z \in A$, then $z \in A \cap B$.

Now assume that $\left\{x_{n}\right\}$ be a sequence in $Y$ such that $\alpha\left(x_{2 n}, x_{2 n+1}\right) \geq 1$ and $\beta\left(x_{2} n, x_{2 n+1}\right) \geq 1$ for all $n \in \mathbb{N} \cup\{0\}$ and $x_{n} \rightarrow x$ as $n \rightarrow \infty$, then $x_{2 n} \in A$ and $x_{2 n+1} \in B$. Since $B$ is closed, and $X$ is an ordered $\alpha$-regular, we have $x \in B$ and $x_{2 n} \leq x$, hence $\alpha\left(x_{2 n}, x\right) \geq 1$ and $\beta\left(x_{2 n}, x\right) \geq 1$. We deduce that all the hypotheses of above Theorems 2.1-2.2 are satisfied with $X=Y$ and hence $T$ has a fixed point.

\section{Related results}

Theorem 3.3 Let $(X, d)$ be a complete metric space, $T$ is self-mapping, $T: X \rightarrow X$, and $\alpha: X \times X \rightarrow \mathbb{R}$. Suppose that the following conditions are satisfied:

(i) $T$ is an $(\alpha)$-admissible mapping;

(ii) T satisfies any one of following rational contractive mapping;

$$
\begin{aligned}
& {[\psi(d(T x, T y))+l]^{\alpha(x, T x) \alpha(y, T y)}} \\
& \quad \leq \theta(\psi(M(x, y))) \psi(M(x, y))+l ;
\end{aligned}
$$

or

$$
\begin{aligned}
& \alpha(x, T x) \alpha(y, T y) \psi(d(T x, T y)) \\
& \quad \leq \theta(\psi(M(x, y))) \psi(M(x, y)),
\end{aligned}
$$

where $M(x, y)=$

$\max \left\{d(x, y), d(x, T x), d(y, T y), \frac{d(x, T x)}{}\right.$

$\left.d(y, T y) 1+d(x, y), \frac{d(x, T x) d(y, T y)}{1+d(T x, T y)}\right\}, \psi \in \Psi$ and $l \geq 1$. (iii) there exists $x_{0} \in X$ such that $\alpha\left(x_{0}, T x_{0}\right) \geq 1$ and $\beta\left(x_{0}, T x_{0}\right) \geq 1$; 
(iv) either $T$ is continuous or $X$ is $\alpha$-regular. Then, $T$ has a fixed point $x^{*} \in X$ and $\left\{T^{n} x_{0}\right\}$ converges to $x^{*}$. Further, if For all $x, y \in F(T)$, with $x \neq y$ such that $\alpha(x, T x) \geq 1$, and $\alpha(y, T y) \geq 1$, then $T$ has $a$ unique fixed point in $X$.

Corollary 3.1 [11] Let $(X, d)$ be a complete metric space and $T: X \rightarrow X$ be an $\alpha$-admissible mapping. Assume that there exists a function $\theta \in \Theta$, such that

$$
(d(T x, T y)+l)^{\alpha(x, T x) \alpha(y, T y)} \leq \theta(d(x, y)) d(x, y)+l
$$

for all $x, y \in X, l \geq 1$. Suppose that either

(a) $T$ is continuous or

(b) if $\left\{x_{n}\right\}$ is a sequence in $X$ such that $x_{n} \rightarrow x$, $\alpha\left(x_{n}, x_{n+1}\right) \geq 1$ for all $n$, then $\alpha(x, T x) \geq 1$.

If there exists $x_{0} \in X$ such that $\alpha\left(x_{0}, T x_{0}\right) \geq 1$, then $T$ has a fixed point.

Corollary 3.2 [11] Let $(X, d)$ be a complete metric space and $T: X \rightarrow X$ be an $\alpha$-admissible mapping. Assume that there exists a function $\theta \in \Theta$, such that

$$
\alpha(x, T x) \alpha(y, T y) d(T x, T y) \leq \theta(d(x, y)) d(x, y)
$$

for all $x, y \in X$. Suppose that either

(a) $T$ is continuous or

(b) if $\left\{x_{n}\right\}$ is a sequence in $X$ such that $x_{n} \rightarrow x$, $\alpha\left(x_{n}, x_{n+1}\right) \geq 1$ for all $n$, then $\alpha(x, T x) \geq 1$.

If there exists $x_{0} \in X$ such that $\alpha\left(x_{0}, T x_{0}\right) \geq 1$, then $T$ has a fixed point.

Theorem 3.4 Let $(X, d)$ be a complete metric space, $T$ is self-mapping, $T: X \rightarrow X$, and $\alpha: X \times X \rightarrow \mathbb{R}^{+}$. Suppose that the following conditions are satisfied:

(i) $T$ is an $\alpha$-admissible mapping;

(ii) $T$ satisfies the following contractive condition

$$
\alpha(x, y) \psi(d(T x, T y)) \leq \theta(\psi(M(x, y))) \psi(M(x, y)) ;
$$

or

$$
[\psi(d(T x, T y))+l]^{\alpha(x, y)} \leq \theta(\psi(M(x, y))) \psi(M(x, y))+l,
$$

where $M(x, y)=\max \{d(x, y), d(x, T x), d(y, T y)$, $\left.\frac{d(x, T x) d(y, T y)}{1+d(x, y)}, \frac{d(x, T x) d(y, T y)}{1+d(T x, T y)}\right\}, \psi \in \Psi$ and $l \geq 1$.

(iii) there exists $x_{0} \in X$ such that $\alpha\left(x_{0}, T x_{0}\right) \geq 1$;

(iv) either $T$ is continuous or $X$ is $\alpha$-regular.

Then, Thas a fixed point $x \in X$ and $\left\{T^{n} x_{0}\right\}$ converges to $x$.

Further, if for all $x, y \in F(T)$, with $x \neq y$ such that $\alpha(x, T x) \geq 1, \alpha(y, T y) \geq 1$, then $T$ has a unique fixed point in $X$.

Proof Define mapping $\beta: X \times X \rightarrow[0, \infty)$ defined as $\beta(x, y)= \begin{cases}1, & \text { if } x, y \in X \\ 0, & \text { otherwise }\end{cases}$

Now using the above Theorems 2.1-2.2, we get the result.

\section{Fixed point results for graphic contractions}

Consistent with Jachymski [12], let (X, d) be a metric space and $\Delta$ denotes the diagonal of the Cartesian product $X \times X$. Consider a directed graph $G$ such that the set $V(G)$ of its vertices coincides with $X$, and the set $E(G)$ of its edges contains all loops, i.e., $E(G) \supseteq \Delta$. We assume $G$ has no parallel edges, so we can identify $G$ with the pair $(V(G), E(G))$. Moreover, we may treat $G$ as a weighted graph (see [12]) by assigning to each edge the distance between its vertices. If $x$ and $y$ are vertices in a graph $G$, then a path in $G$ from $x$ to $y$ of length $m(m \in \mathbb{N})$ is a sequence $\left\{x_{i}\right\}_{i=0}^{m}$ of $m+1$ vertices such that $x_{0}=x, x_{m}=y$ and $\left(x_{i-1}, x_{i}\right) \in E(G)$ for $i=1, \ldots, m$. A graph $G$ is connected if there is a path between any two vertices. $G$ is weakly connected if $\tilde{G}$ is connected (see for details $[3,12]$ ). Recently, some results have appeared providing sufficient conditions for a mapping to be a Picard operator if $(X, d)$ is endowed with a graph. The first result in this direction was given by Jachymski [12].

Definition 3.1 [12] Let $(X, d)$ be a metric space endowed with a graph $G$. We say that a self-mapping $T: X \rightarrow X$ is a Banach $G$-contraction or simply a $G$-contraction if $T$ preserves the edges of $G$, that is, $(x, y) \in E(G) \Longrightarrow(T x, T y) \in$ $E(G)$ for all $x, y \in X$ and $T$ decreases the weights of the edges of $G$ in the following way:

$\exists \alpha \in(0,1)$ such that for all $x, y \in X,(x, y) \in E(G) \Longrightarrow$ $d(T x, T y) \leq \alpha d(x, y)$.

Theorem 3.5 Let $(X, d)$ be a complete metric space endowed with a graph $G, T$ is a self-mapping, $T: X \rightarrow X$, satisfying

$$
\psi(d(T x, T y)) \leq \theta(\psi(M(x, y))) \psi(M(x, y)) ;
$$

or

$$
\psi(d(T x, T y))+l \leq \theta(\psi(M(x, y))) \psi(M(x, y))+l,
$$

where $\quad M(x, y)=\max \{d(x, y), d(x, T x), d(y, T y), \underline{d(x, T x)}$ $\left.d(y, T y) 1+d(x, y), \frac{d(x, T x) d(y, T y)}{1+d(T x, T y)}\right\}, \psi \in \Psi$ and $l \geq 1$.

Suppose that the following assertions hold:

(i) for all $x, y \in X, \quad(x, y) \in E(G)$, implies $(T x, T y) \in E(G)$

(ii) $\quad\left\{x_{n}\right\}$ is a sequence in $X$ such that $x_{n} \rightarrow x$ as $n \rightarrow$ $\infty$ and $\left(x_{n}, T x_{n}\right) \in E(G)$, then $(x, T x) \in E(G)$;

(iii) there exists $x_{0} \in X$ such that $\left(x_{0}, T x_{0}\right) \in E(G)$. 
Then, $T$ has a fixed point $x \in X$.

Proof Define mapping $\alpha: X \times X \rightarrow[0, \infty)$ defined as $\alpha(x, y)= \begin{cases}1, & \text { if } \quad(x, y) \in G, \\ 0, & \text { otherwise }\end{cases}$

Now, we show that $T$ is an $\alpha$-admissible mapping. Suppose that $\alpha(x, y) \geq 1$. Therefore, we have $(x, y) \in E(G)$. From (i), we get $(T x, T y) \in E(G)$. So, $\alpha(T x, T y) \geq 1$ and $T$ is an $\alpha$ admissible mapping. Hence, from the definition of $\alpha$ and inequality, we have

$$
\alpha(x, y) \psi(d(T x, T y)) \leq \theta(\psi(M(x, y))) \psi(M(x, y))
$$

or

$$
[\psi(d(T x, T y))+l]^{\alpha(x, y)} \leq \theta(\psi(M(x, y))) \psi(M(x, y))+l,
$$

where $\quad M(x, y)=\max \{d(x, y), d(x, T x), d(y, T y)$, $\left.\frac{d(x, T x) d(y, T y)}{1+d(x, y)}, \frac{d(x, T x) d(y, T y)}{1+d(T x, T y)}\right\}, \psi \in \Psi$ and $l \geq 1$.

From (iii) there exists $x_{0} \in X$ such that $\left(x_{0}, T x_{0}\right) \in E(G), \alpha\left(x_{0}, T x_{0}\right) \geq 1$. Let $\left\{x_{n}\right\}$ is a sequence in $X$ such that $x_{n} \rightarrow x$ as $n \rightarrow \infty$ and $\left(x_{n}, T x_{n}\right) \in E(G)$ for all $n \in \mathbb{N}$, then $\alpha\left(x_{n}, T x_{n}\right) \geq 1$. Thus, from (ii) we get, $(x, T x) \in E(G)$. That is, $\alpha(x, T x) \geq 1$. Therefore, all conditions of Theorem 3.4 hold true and $T$ has a fixed point.

Remark 3.1 Using the technique of Samet et al. [15], we can obtain corresponding coupled fixed point results from our Theorems 2.1, 2.2.

Acknowledgments The author is thankful to the learned referees for very careful reading and valuable suggestions.

Open Access This article is distributed under the terms of the Creative Commons Attribution 4.0 International License (http:// creativecommons.org/licenses/by/4.0/), which permits unrestricted use, distribution, and reproduction in any medium, provided you give appropriate credit to the original author(s) and the source, provide a link to the Creative Commons license, and indicate if changes were made.

\section{References}

1. Banach, S.: Sur les opéerations dans les ensembles abstraits et leur application aux équations integrales. Fund. Math. 3, 133-181 (1922)

2. Berzig, M., Rus, M.-D.: Fixed point theorems for $\alpha$-contractive mappings of Meir-Keeler type and applications. Nonlinear Anal. Model. Control. 19(2), 178-198 (2014)

3. Bojor, F.: Fixed point theorems for Reich type contraction on metric spaces with a graph. Nonlinear Anal. 75, 3895-3901 (2012)

4. Chandok, S., Choudhury, B.S., Metiya, N.: Some fixed point results in ordered metric spaces for rational type expressions with auxiliary functions. J. Egypt. Math. Soc. 23(1), 95-101 (2015). doi:10.1016/j.joems.2014.02.002

5. Chandok, S., Khan, M.S., Abbas, M.: Common fixed point theorems for nonlinear weakly contractive mappings. Ukrainian Math. J. 66(4), 531-537 (2014)

6. Chandok, S., Khan, M.S., Narang, T.D.: Fixed point theorem in partially ordered metric spaces for generalized contraction mappings. Azerbaijan J. Math. 5(1), 89-96 (2015)

7. Chandok, S., Postolache, M.: Fixed point theorem for weakly Chatterjea type cyclic contractions. Fixed Point Theory Appl. 2013, 28 (2013) doi:10.1186/1687-1812-2013-28

8. Geraghty, M.: On contractive mappings. Proc. Am. Math. Soc. 40, 604-608 (1973)

9. Amini-Harandi, A., Emami, H.: A fixed point theorem for contraction type maps in partially ordered metric spaces and application to ordinary differential equations. Nonlinear Anal. 72, 2238-2242 (2010)

10. Harjani, J., Sadarangani, K.: Fixed point theorems for weakly contractive mappings in partially ordered sets. Nonlinear Anal. 71, 3403-3410 (2009)

11. Hussain, N., Karapinar, E., Salimi, P., Akbar, F.: $\alpha$-admissible mappings and related fixed point theorems. J. Inequal. Appl. 2013, 114 (2013)

12. Jachymski, J.: Equivalent conditions for generalized contractions on (ordered) metric spaces. Nonlinear Anal. 74, 768-774 (2011)

13. Karapinar, E.: $\alpha-\psi$-Geraghty contraction type mappings and some related fixed point results. Filomat 28(1), 37-48 (2014)

14. La Rosa, V., Vetro, P.: Common fixed points for $\alpha, \psi, \phi$-contractions in generalized metric spaces. Nonlinear Anal.: Model. Control 19(1), 43-54 (2014)

15. Samet, B., Vetro, C., Vetro, P.: Fixed point theorem for $\alpha-\psi$ contractive type mappings. Nonlinear Anal. 75, 2154-2165 (2012) 